\title{
A Model for E-commerce Market Network with Improved Evolution Mechanism
}

\author{
Zhihong TIAN ${ }^{1}$, Zhenji ZHANG ${ }^{1}$, Xiaolan GUAN ${ }^{2}$ \\ ${ }^{1}$ Beijing Jiaotong University, Beijing, 100044, China, \\ 10113130@bjtu.edu.cn \\ ${ }^{2}$ Beijing Institute of Graphic Communication, Beijing, 102600, China
}

\begin{abstract}
In order to investigate the formation of e-commerce market network, this paper describes an analytical framework from a complex network point of view which contains three steps-definition of network, analysis of network topology and analysis of network environment. Then, an innovative model for explaining the evolutionary process is proposed, with several original factors-growth-factor, select-order-factor, preferential attachment mechanism and global-local-factor. Our research reveals that the attraction mechanism impacts evolutionary trend and network structure to some extent, and also reveals that the global-local-factor and select-order-factor impact the evolutionary structure of the network, the smaller the probability, the smaller the concentration of networks and the more obvious the randomness are. In order to analyze the impact of edge-increasing mechanism on network evolutionary trend, a contrast test is designed with two models. The test reveals that the edge-increasing mechanism makes the network become a small world with a lower average distance and a higher clustering coefficient, and makes it be like a scale-free network with a lower power-law exponent and a higher centralization.
\end{abstract}

Keywords: E-commerce; Market network; Evolution model; Complex network, Scale-free network.

\section{Introduction}

Significant progress has been made in e-commerce applications so far, and e-commerce plays a very important role in national economy. Large numbers of buyers and sellers interact with each other through transactions on websites. These interactions promote the evolution and shape complex structures of e-commerce market. Going deeply insight into research of e-commerce market network has a profound and lasting significance.

Social and business networks are an increasingly important area of research attention in many disciplines [1-3]. However, stable equilibriums and models have been mainly focused on, while their dynamics and evolution have received limited research attention. One of the major challenges is to better understand, predict and control their dynamics, including how they form, evolve and shape their behaviors and performances [4][5]. Networks of relationships, such as the nature, development and evolution of firms and markets, in all business disciplines have been drawn attention, rather than being attributed only to individual characteristics. In marketing, the research about B2B markets in the US, Europe and Australasia is a pioneer [6].

The market-as-networks (MAN) perspective is a theoretical framework that aims to describe and understand the functioning of industrial markets. It perceives industrial markets as a network of interdependent relationships between companies that cooperate, compete, compete to cooperate with each other and cooperate in order to compete more effectively [7]. Some particular characteristics of e-commerce market have been studied. Maurer $\mathrm{SM}$, et al. [8] present a static competition model of web site growth by establishing prey-predator equations. Their research shows that, in the long term, under competitive conditions, an efficient equilibrium structure will somehow emerge and, if conditions change leading to another equilibrium point, the network will move to that equilibrium in the long run. However, there are several troubles in the evolution process of e-commerce market network. Firstly, we do not know how long the long run is, whether a network will ever get to such an equilibrium point. The evolution process is a black box for us. Secondly, we do not know what the network looks like. Namely, a clear and intuitive description of network structure has not been provided. Thirdly, we do not know how the network changes when its evolutionary rules or parameters change.

The aim of the paper is to propose a complex network analytical framework for e-commerce market networks, and to analyze the forming mechanisms. Then, a new evaluating model with growth-factor, select-order-factor, preferential attachment mechanism and global-local-factor is built. Our research reveals that the attraction mechanism impacts evolutionary trend and 
network structure to some extent. Contrast to the evolutionary trend of degree preferential attachment mechanism, the evolutionary trend with attraction preferential attachment mechanism reaches a smaller power-law exponent generally. Our research also reveals that the global-local-factor and select-order-factor impact the evolutionary structure of the network, and the smaller the probability is, the smaller the concentration of networks is, and the more obvious the randomness is. In order to analyze the impact of edge-increasing mechanism on network evolutionary trend, a contrast test is designed with two models. The test reveals that the edge-increasing mechanism makes the network become a small world with a lower average distance and a higher clustering coefficient, and makes it be like a scale-free network with a lower power-law exponent and a higher centralization.

\section{An Analytical Framework for E-commerce Market Networks}

Complex network theory is a new theory that synthesizes the research results of self-organization theory, nonlinear theory and complexity theory in the past. In the complex network theory, it has been discovered that there is a significant relationship between the behavior of agents and their underlying network structures. As the structure and morphology of complex systems, it focuses on the topological characteristics of the system structure. We can put any complex system as a complex network to study. Using complex network to study complex system can enhance the in-depth understanding of system structure. Hence, we present a network analysis framework for e-commerce market.

The network is constituted by nodes and links between nodes (edges). Nodes represent the elements in a complex system, and the edges represent the interaction between system elements. E-commerce market is a complex network, and its complexity reflects in the following areas:

(1) The system has a huge number of nodes, and its network structure is complex and presents a variety of different characteristics. There are the generation and disappearance of nodes or edges. The emergence and demise of elements that have the life-cycle characteristic is very common. The relationships among elements are also dynamic changed.
(2) Networks are no governance structures. There is not one dominant organization to control and organize the other members in the network. Rather, the network concept is a way to visualize and understand the way firms and organizations are interconnected directly and indirectly through relationships. Networks are not under the control of individual nodes.

(3) Networks are formed in a self-organizing way through the actions and interactions of actions and interactions of actors involved, as they occur over time. They are continually being made and remade (or not) through ongoing structuring and restructuring processes. The multiple interactions and feedback effects continually taking place in networks lead to a complexity that makes it very difficult to control and predict for any individual actor.

(4) A business network does not have clear boundaries or a dominant centre. The scope of network depends on specific research perspective and question. For example, all individuals and organizations relating to e-commerce can be involved into a huge and complex network. However, in order to reduce the difficulties of research and simplify models, network's scope should be determined first. E-commerce enterprises and customers can compose the B2C network. Enterprises which are in the same value chain can compose B2B network. A big enterprise and its cooperators compose a community network, and so on. The network perspective on e-commerce marketing has wide-ranging implications for understanding the behaviors, the opportunities and strategies available to participants in the network. The structure of the network is both a limiting and enabling factor for individual firms.

(5) The macro environment includes the social-cultural space in which businesses operate. Businesses depend on the level of knowledge and the institutional framework, the legal institutions and moral norms. Social-cultural norms and laws set includes various rules for behavior that affect interactions between firms and the kinds of relations that can be formed and not formed. These include contractual, property rights and trade practices acts. Also, the development of specialization and division of labor will have important externalities through its impacts on people's lives, the environment, social institutions, politics and power, religious and other values, as well as social life in general. 
(6) Time plays a central role in explaining and understanding exchange. Business relations develop over time and they are path-dependent. Buyers and sellers actively take into account what has happened before and they also form plans and have expectations of what is likely to happen in the future, both of which affect their decisions in the present. The state of the network subject (person or organization) changes over time.

From a mathematical point of view, network can be described by set $\boldsymbol{N}=\{\boldsymbol{V}, \boldsymbol{E}\}$ composed with nodes set $\boldsymbol{V}=\left\{v_{1}, v_{2}, \ldots, v_{n}, \ldots\right\}$ and edges set $\boldsymbol{E}=\left\{e_{1}, e_{2}, \ldots, e_{n}, \ldots\right\}$.

Network analysis framework for e-commerce market is as the following Figure. 1. From the graph we can see that, there are three steps for analyzing on e-commerce market from a network point of view-definition of network, analysis of network topology and analysis of network environment. Next, the first two steps will be discussed in detail.

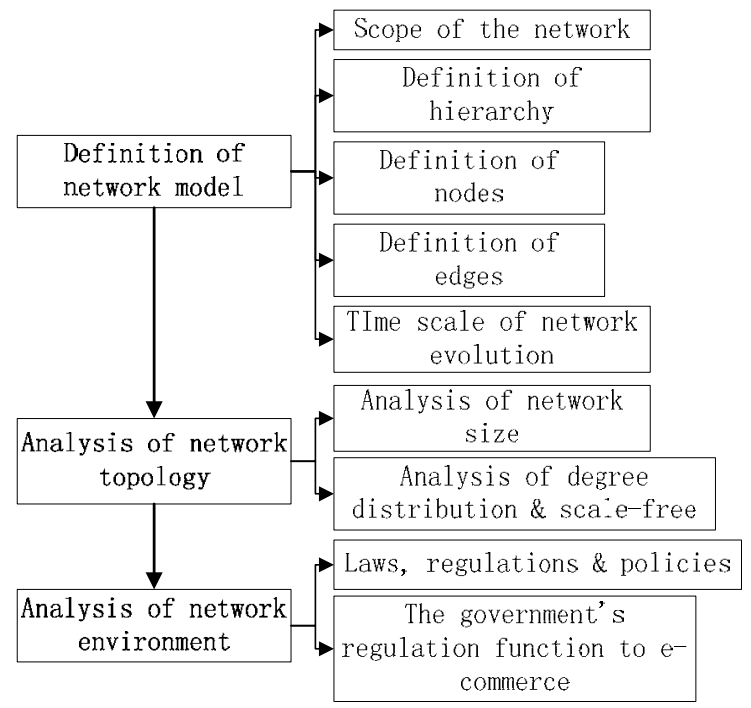

Figure 1. E-commerce network analysis framework

\subsection{Definition for network model}

\section{Scope of the network}

In general, according to different research purposes, we can have different definitions of the scope of e-commerce. The generalized scope covers all trading business activities through electronic communication technology, including e-commerce service providers, e-commerce users, independent e-commerce enterprises and social business enterprise, and so on. In addition to the "generalized scope", there is another kind of e-commerce market network scope - enterprise e-commerce network with one or several leading enterprises as the core. For example, Alibaba group is a typical enterprise network.

\section{Definition for hierarchy}

From the perspective of ecological system, e-commerce market network can simply be divided into three levels - individual, population and community. Individuals are enterprises, organizations, consumers, or other entities. Population contains all similar individuals sharing a same gene pool, such as business model, organizations with similar purpose, and groups of consumer with similar demands, etc. Community is the sum of all species in a certain environment.

\section{Definition for nodes}

Different definition of node set will essentially affect the size and density of network, and then ultimately affect the subsequent analysis. Therefore, node set should contain all of the distinguished individuals in interaction and change with the scope and level of research changing. In addition, the definition of nodes should be kept consistent in cross-network researches. In e-commerce market network, the main kinds of nodes include B2B website nodes, B2C website nodes, personal web merchants and customers. B2B competition network contains only one type of nodes (business website) which is called 1-mode network, and B2C network normally contains two types of nodes (business website and customer) which is called 2-mode network. However, in e-commerce platform ecosystem network, there could be more types of nodesthe core e-commerce companies, suppliers, customers, financial institutions, certification bodies, payment institutions, and logistics companies, etc.

\section{Definition for edges}

There are two main relationships between each pair of linked node - competition and collaboration. Because of the limit of resources and the overlap of enterprise niches, competition is eternal. Competition promotes the development of system towards diversification and non-equilibrium, which are the important conditions of self-organization evolution. Competition and cooperation are mutually contradictory and interdependent. Competition leads to diversification while collaboration promotes division of labor and 
cooperation, and leads to niche markets and innovation modes.

\section{Time scale of network evolution}

Time scale is the average measurement of involved time for a physical process. In general, the slower the physical process is, the longer the time scale is. The larger the involved space of physical processes is, the longer the time scale is. Economic data is usually released with a time horizon of months, quarters and years. According to different research problems, different time scales should be used. In the research of evolution of e-commerce market, time scales are months or years in general.

\subsection{An approach to e-commerce market network topology}

\section{Analysis of network size}

Analysis of network size is cumulating the number of nodes in network and recording the changes in the time axis. From 1997 to 2012, the number of e-commerce enterprises is going up. However, there are several stages with different acceleration. From 2000 to 2003, due to the burst of Internet bubble, e-commerce market has an immense downward momentum, and business expansion has slowed. In the year of 2003, the market gradually got out of the recession. Meanwhile, SARS accelerated the popularization of electronic commerce. From 2006 to 2007, most B2B websites began to profit, and developed more healthily. Since 2008, traditional industries tried to enter e-commerce market, and lots of specialized and segmented enterprises were born. More abundant service pattern and profit pattern appeared, and the network scale expanded in an unprecedented speed [9].

\section{Analysis of degree distribution \& scale-free}

The spread in the number of edges of a node, or node degree, is characterized by a distribution function $P(k)$, which describes the probability that a randomly selected node $i$ has exactly $k_{i}$ edges. Emergence of a power-law in the degree distribution $P(k) \sim k^{-\gamma}$ in complex networks is an interesting self-organized phenomenon in complex systems. Such a network is called scale-free. In marketing, meanings of degree distribution vary depending on different network structure hypotheses. In general, two indexes-market trading volume and user penetration are measured to reflect the e-commerce market share situation. From
Figure 2 and Figure 3 we can see that the degree distribution of e-commerce market network follows power-law.

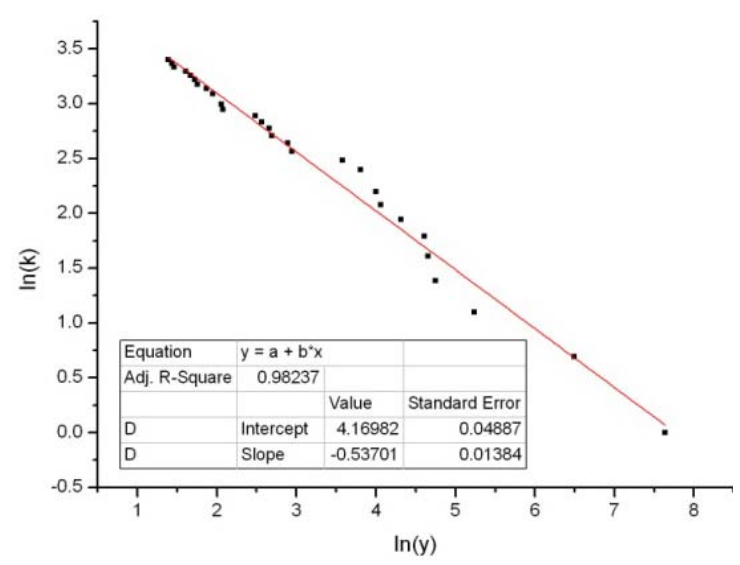

Figure 2. Log-log coordinate of market trading volume in B2B websites

(Data source: IRESEARCH [10])

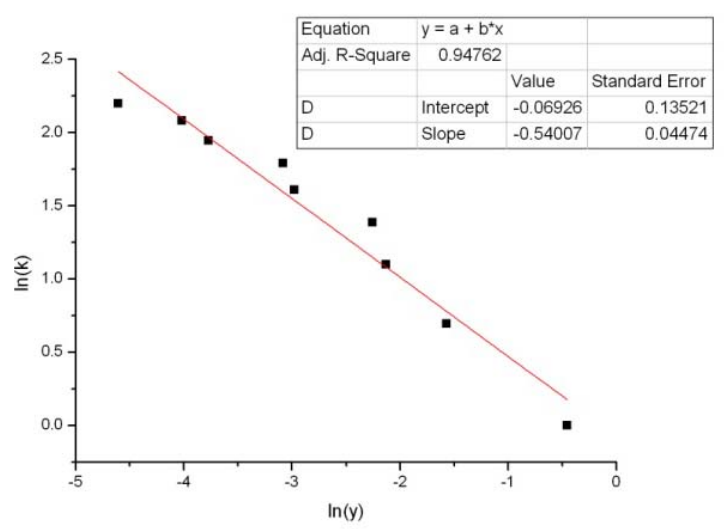

Figure 3. Log-log coordinate of user penetration of B2C websites (Data source: CNNIC [11])

\section{Mechanisms of e-Commerce Network Formation and Modelling}

\subsection{Metabolism mechanisms}

An evolving system has the only way metabolism to maintain its existence and structure. Because of the entropic-adding process, system must constantly exchange material, energy and information with the external environment. Therefore, growth and death are the basic mechanisms in evolution process. In e-commerce market, at any time, some businesses are born, while others die. The mortality rate in Internet market is significantly higher than that in traditional market since special features of Web economic. 
A typical case is the development of group-buying websites in China from 2010 to now. According to the report published by China E-Commerce Research Center, the number of surviving group-buying websites now has deduced to less than 2000 from the peak 5188 in July 2011. The nationwide survey shows that a total of 6218 group-buying websites have been born, and 4670 ones have already died due to fierce competition.

\subsection{Adaptation mechanisms}

In order to adapt to network, economic actors have to establish and maintain a relationship through their interactions with each other over time. In this process, there are two crucial mechanisms - search mechanism and selection mechanism.

\section{Search mechanism}

The first step of relationship initiation concerns the awareness of prospective exchange partners. Searching for a new exchange partner is a goal-oriented activity, initiated by an actor to improve its access to certain resources or capabilities. Relationships can be initiated through the actors' existing personal and professional networks, or through advertising and communication efforts. Therefore, in the evolution model, local and global search scope should be both considered. When an economic actor realizes shortcomings regarding resources or capabilities that it currently does not control, it will initiate a deliberate search process for potential suppliers.

\section{Selection mechanism}

In past research works, there are two typical selection mechanisms - rich-get-rich and fit-get-richer. The rich-get-rich rule is implemented by newcomers preferential connecting to old nodes with certain topological characteristics [12]. In fact, this extreme assumption is not always available for many networks when their sizes are huge. The second one is the fit-get-richer mechanism. In our research, a new rule named "attractive-get-rich" is proposed. Attraction for economic actors is predominantly derived as net value that can be gained through a relationship, i.e. the costs and benefits for each party in the relationship in short and long term. In addition, because of huge cost of information acquisition and processing, businesses can't investigate and select the optimal cooperator from all potential nodes in the whole network. Besides shrink of the scope, there is inevitable randomness in selection process. Therefore, random variable should also be introduced in the selection process with "attractive - get - rich" together.

\subsection{Modelling}

In the network model, the link structure of the network is defined by the adjacency matrix $a_{i j}$, in which $a_{i j}=1$ if a link between the nodes $i$ and $j$ exists and 0 otherwise. The model starts from an initial $m_{0}$ complete graph and each node $i$ is endowed with an fitness $x_{i} \geq 0$, drawn from a given probability distribution $f(x)$. The two ingredients-growth and preferential attachment, inspire the introduction of the scale-free model that leads to a network with a power-law degree distribution. The algorithm of the scale-free model is the following [12]:

(1) Growth: Starting with a small number $\left(m_{0}\right)$ of nodes, at every time step we add a new node with $m\left(\leq m_{0}\right)$ edges that link the new node to $m$ different nodes already present in the system.

(2) Preferential attachment: When choosing the nodes to which the new node connects, we assume that the probability $\Pi$ that a new node will be connected to node $i$ depends on the degree $k_{i}$ of node $i$, such that

$$
\Pi=k_{i} / \sum_{n} k_{i}
$$

In fact, this extreme assumption is not always available for many networks when their sizes are huge. Based on BA model, a revised model is proposed with following mechanisms growth-factor, select-order-factor, preferential attachment mechanism and global-local-factor.

\section{Growth-factor}

We define a random growth-factor $p_{1}\left(p_{1} \in\right.$ $[0,1])$ to indicate the growth or death of the network in each time step.

$$
\left\{\begin{array}{c}
p_{1}, \text { growth } \\
1-p_{1}, \text { death }
\end{array}\right.
$$

Add a node in a probability $p_{1}$. At every time step, a new node enters the network with $m(\leq$ $m_{0}$ ) edges that link the new node to $m$ different nodes. Delete a node in a probability $1-p_{1}$. Which node should be deleted depends on the counter-preferential attachment. 


\section{Select-order-factor}

Selection mechanisms indicate which nodes should be linked when a new node is entering the network. There are two extremes - random selection and preferential selection. In real world, the two mechanisms coexist. Hence, we define a select-order-factor $p_{2}\left(p_{2} \in[0,1]\right)$ to indicate the selection mechanism in each time step.

$\left\{\begin{array}{l}p_{2}, \text { preference } \\ 1-p_{2}, \text { random }\end{array}\right.$

\section{Attraction attachment mechanism}

When a new coming node decides which old node to connect, a probability $\Pi_{i}$ is assumed. Whether a new node will connect to node $i$ depends on the attraction $a t t_{i}$ of node $i . a t t_{i}=g\left(k_{i}\right.$, $\left.x_{i}\right)$. If $a t t_{i}=k_{i}$, the preferential attachment mechanism is equal to BA model. $x_{i}$ is a fitness $\mu_{i}$ which follows a given probability $f(\mu)$.

$\Pi_{i}=a t t_{i} / \sum_{n} a t t_{i}$

\section{Global-local-factor}

The global preferential selection probability is $p_{3}$ $\left(p_{3} \in[0,1]\right)$. We define the global-local-factor $p_{3}$ to indicate the selection range in each time step.

$\left\{\begin{array}{c}p_{3}, \text { global } \\ 1-p_{3}, \text { local }\end{array}\right.$

The size of local world in which all nodes are randomly selected from global network is defined as variable $M$.

\section{Simulation Results and Analysis}

All simulation are initiated with an initial network consisting of nodes $\left(m_{0}=3\right)$. It is also assumed that one node enters the network with $m(m=3)$ links or leaves there at one time unit. For our simulation runs, we controlled several variables.

\subsection{Model validation tests}

Test 1: The validation of basic random selection mechanism

The basic random selection mechanism is simulated to check whether our simulation code is valid. In the test, we set $p_{1}=1$ (the network always increases), $p_{2}=0$ (random selection), $p_{3}=1$ (global selection), and the evolution time units $N=1000$. The simulation result matches prior research one that the random selection results in an exponential distribution. The degree distribution of the simulation result is shown in Figure. 4. The regression $R^{2}$ is 0.996, which is significantly high. We can conclude that the model is valid for basic random selection mechanism.

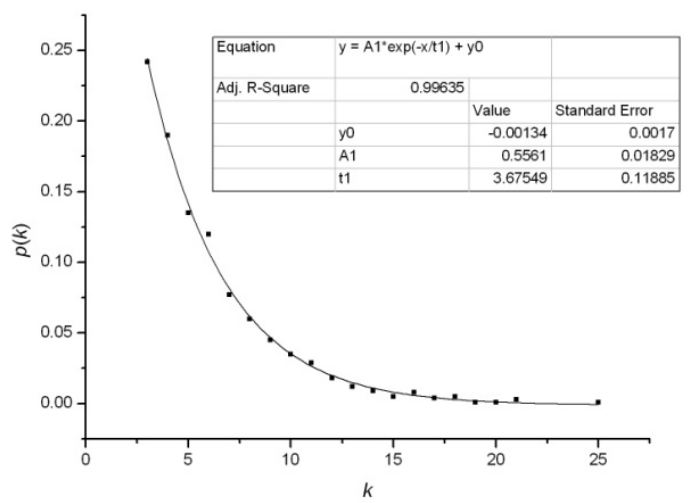

Figure 4. The degree distribution $p(k)\left(m_{0}=3, m=3\right.$,

$$
\left.p_{1}=1, p_{2}=0, p_{3}=1\right)
$$

Test 2: The validation of basic preferential selection mechanism

The second test is to exam the valid of the basic preferential selection mechanism. In the test, we set $p_{1}=1$ (the network always increases), $p_{2}=1$ (preferential selection), $\quad p_{3}=1$ (global selection), and the evolution time units $N=1000$. The simulation result matches prior research one, which proves empirically and theoretically that the exponent $\gamma$ of the power law is between 2 and 3 . The degree distribution of our simulation result is shown in Figure. 5. The regression of the distribution shows that its exponent $\gamma$ is about 2.9 and the regression $R^{2}$ is 0.994 , which is significantly high. We can conclude that the model is valid for basic preferential selection mechanism.

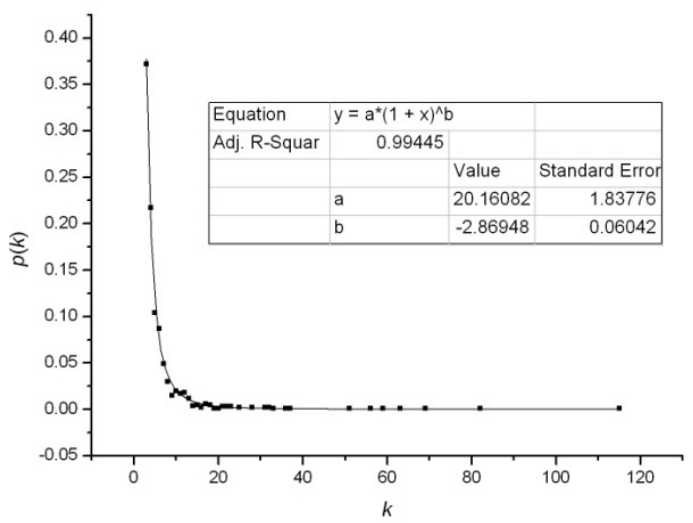

Figure 5. The degree distribution $p(k)\left(m_{0}=3, m=3\right.$,

$$
\left.p_{1}=1, p_{2}=1, p_{3}=1\right)
$$

Test 3: The validation of evolutionary stability

The third test is about evolutionary stability. In order to check whether the evolutionary pattern 
is stable near the point of time of observation, the history of the network evolution is traced until the iteration of the preferential attachment (degree preferential attachment) reaches 1000 time units. After a short period of oscillation, the exponents $\gamma$ enter a relatively stable development stage, with the value between 2 and 3 from 200 time unit to 1000 time unit. Figure. 6 shows the evolutionary pattern of 5 times tests.

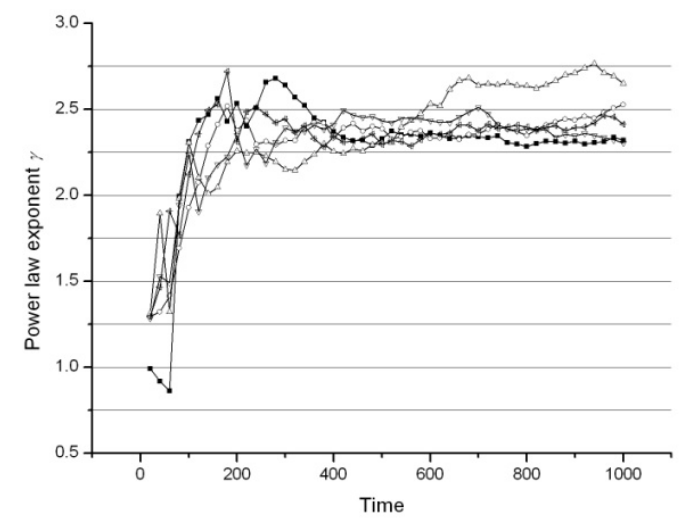

Figure 6. The trend of $\gamma$ of the scale-free network for each 20 time units until 1000 time units $\left(m_{0}=3, m=3\right.$, $p_{1}=1, p_{2}=1, p_{3}=1$ ) (degree preferential attachment)

\subsection{Evolutionary trend of the model with changing variables}

\section{Attraction preferential attachment}

In order to illustrate the impact of attraction on the evolutionary trend, fitness is combined with the degree of nodes. Namely, the preferential attachment rule includes the adaptive and degree of node. The probability $\Pi_{i}$ is as follow:

$$
\Pi_{i}=\left(k_{i}+1\right) \eta_{i} / \sum_{n}\left(k_{i}+1\right) \eta_{i}
$$

The exponent $\gamma$ fluctuates between 2 and 2.6 from 200 time unit to 1000 time unit. Figure. 7 shows the evolutionary pattern of 5 times tests. Contrast to the evolutionary trend of degree preferential attachment mechanism, the evolutionary trend with attraction preferential attachment mechanism reaches a smaller power-law exponent generally. It implies that the majority of relationships are gathered to a few nodes, and the concentration of the network is more obvious. In other words, attraction impacts evolutionary trend and network structure to some extent.

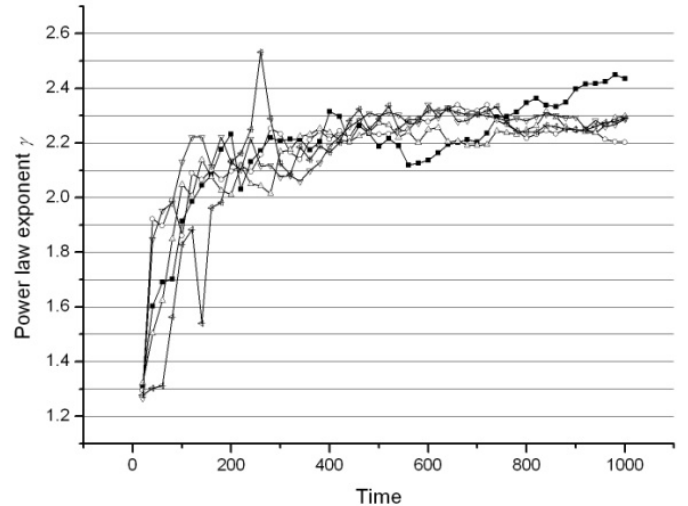

Figure 7. The trend of $\gamma$ of the scale-free network for each 20 time units until 1000 time units $\left(m_{0}=3, m=3\right.$, $p_{1}=1, p_{2}=1, p_{3}=1$ ) (fitness preferential attachment)

\section{Growth-factor}

For finding out the effect of the growth-factor $p_{1}$ on the evolutionary trend of a network, we simulate a network that evolves from an initial network size of 3 nodes for 500 time steps $\left(N=500, p_{2}=1, p_{3}=1\right)$. We run simulations for a variety of different growth rates: $p_{1}=1,0.9,0.8$, and 0.7. The results show that scales of networks vary depending on the differences of growth rates (Figure.8).

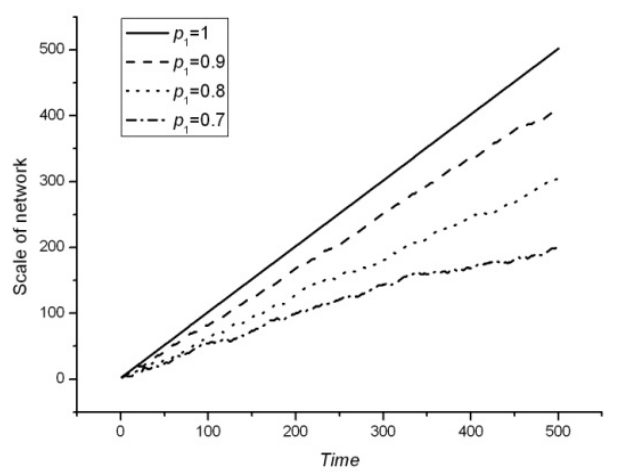

Figure 8. The scales of network for different growth rates

\section{Global-local-factor}

The global-local-factor contains two variable parameters-global selection probability $p_{3}$ and locality size $M$. When the probability $p_{3}$ is 1 , options from which new nodes select extend to the entire network. When the probability $p_{3}$ is 0 , options shrink to a set with $M$ nodes. We design 4 tests for $p_{3}=1,0.7,0.3,0$, and at the same time, $p_{1}=0.9, p_{2}=1$ and $M=3$. After 4000 time intervals, the degree distributions in log-log coordinate are shown in Figure.9. From the graph we can see that, when the probability is 1 , the line connecting all nodes approximates a straight line. However, with the decrease of the probability, the head and tail of the line gradually close to the origin of coordinate, and 
the line approximates a curve. We can conclude that the probability $p_{3}$ impacts the evolutionary structure of the network, and the smaller the probability is, the smaller the concentration of networks is, and the more obvious the randomness is.

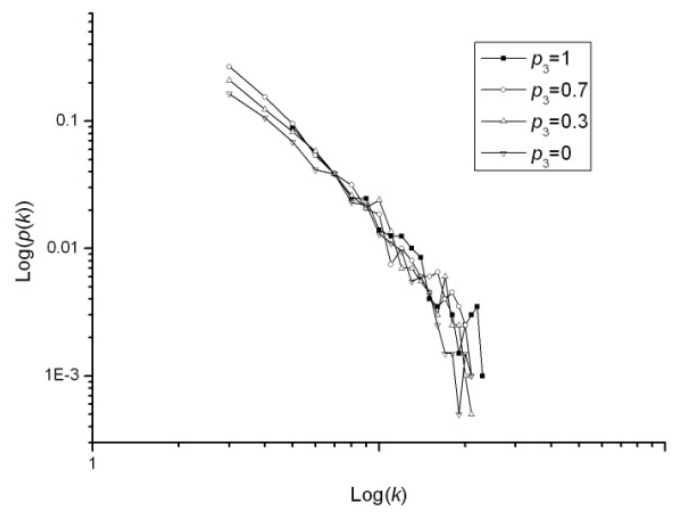

Figure 9. Log-log coordinate of 4 tests with global selection probability

Now we analyze the influence of locality size on network evolution. We design 4 tests for $M=3,10,20$, total nodes number, at the same time, $p_{1}=0.9$, and $p_{3}=0$. After 4000 time intervals, the degree distributions in log-log coordinate are shown in Figure.10. From the graph we can see that, with the decrease of $M$, the head and tail of the line gradually move to the outside. When $M$ is the total number of all nodes, the line approximates a straight line. Hence, the conclusion is that the locality size impacts the evolutionary structure of the network, and the larger the size is, the larger the concentration of networks is, and the more obvious the scale-free is.

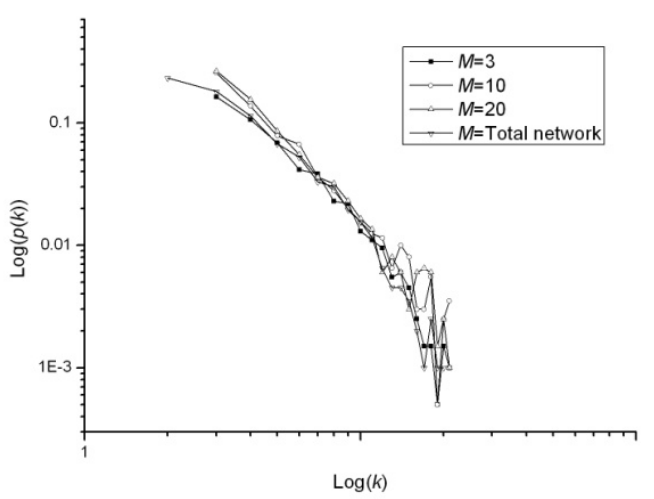

Figure 10. Log-log coordinate of 4 tests with locality size

\section{Select-order-factor}

This section we analyze the influence of select-order-factor $p_{2}$ on network evolution. We design 4 tests for $p_{2}=1,0.7,0.3,0$, at the same time, $p_{1}=0.9$, and $p_{3}=1$. After 4000 time intervals, the degree distributions in log-log coordinate are shown in Figure.11. From the graph we can see that, when the probability is 1 , the line connecting all nodes approximates a straight line. However, with the decrease of the probability, the head and tail of the line gradually close to the origin of coordinate, and the middle moves to the outside, and the line approximates a curve. Hence, the conclusion is that the select-order-factor impacts the evolutionary structure of the network, and the smaller the probability is, the more obvious the randomness is.

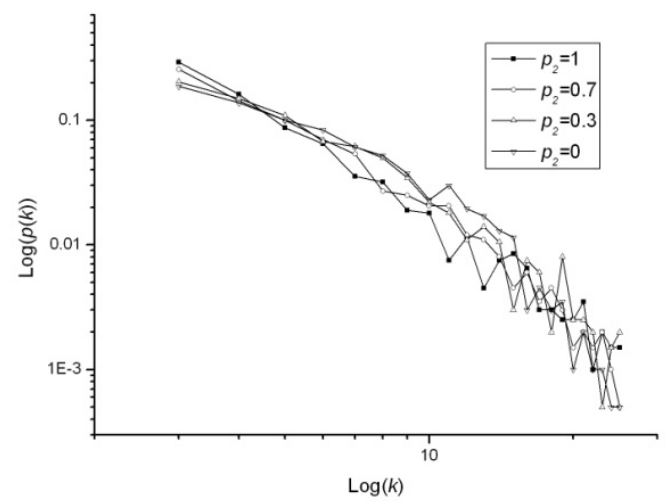

Figure 11. Log-log coordinate of 4 tests with select-order-factor

\subsection{Evolutionary trend of the model with edge-increasing mechanism}

Some relationships are built when enterprise nodes enter the network. And then with the development and demand changing, the above relationships are inevitable adjusted. In this section, we investigate the impact of edge-increasing mechanism on network evolutionary trend. In order to analyze the impact, a contrast test is designed with two models. The model 2 has edge-increasing mechanism and the compared model 1 does not have. We simulate a network that evolves from an initial network size of 3 nodes for 500 time steps $\left(N=500, p_{1}=0.9, p_{2}=1, p_{3}=1\right)$. At each time interval, a link is added into the network with one end selected randomly and the other end selected preferentially. A snapshot is recorded every 100 units.

\section{Mean of degree and number of nodes}

The changing of mean of degree and number of nodes are shown in Figure.12. Obviously, the two indexes of model 2 are both higher than that of model 1. 


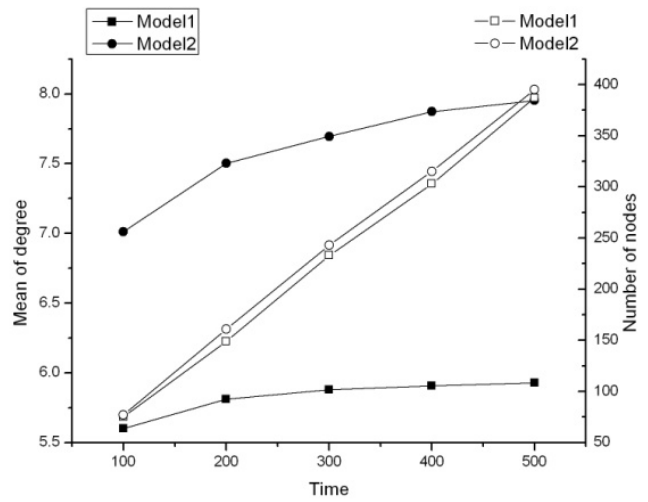

Figure 12. The changing of mean of degree and number of nodes

\section{Clustering coefficient and density of network}

The global clustering coefficient is based on triplets of nodes. A triplet consists of three nodes that are connected by either two (open triplet) or three (closed triplet) undirected ties [13]. A triangle consists of three closed triplets, one centered on each of the nodes. Formally, it has been defined as [13]:

$C=\frac{3 \times n}{N}$

$n$ represents the number of triangles, and $N$ represents the number of connected triples of vertices.

Network density means the degree of interaction of groups' members. From the Figure 13 we can conclude that, the edge-increasing mechanism obviously impacts the clustering coefficient and density of network.

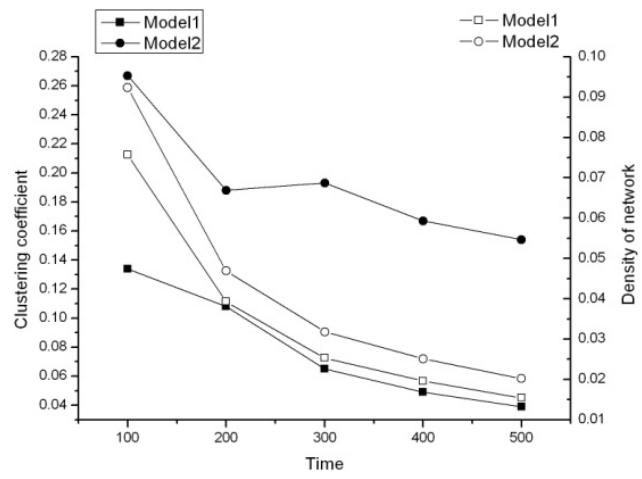

Figure 13. Clustering coefficient and density of network

\section{Average distance and network centralization}

The minimal path length is the shortest path between two nodes (also called geodesic distance) [14][15]. The average distance is the average of all the minimum path lengths between all pairs of nodes in a network. From Figure.14, the average distance is higher of model 1 than that of model 2. The edge-increasing mechanism makes the network be like a small world. And the changing of network centralization is more complex. The network centralization of model 1 is always declining, while that of model 2 is falling first but up then. The edge-increasing mechanism makes the network centralization increase.

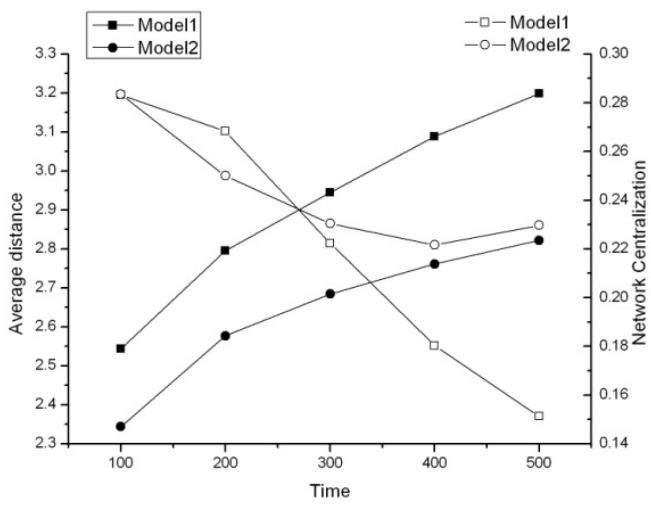

Figure 14. Average distance and network centralization

\section{Power law exponent}

The Figure.15 shows the evolutionary trends of power law exponents of two models. Relative to model 1, the power law exponent is obviously smaller. Namely, the edge-increasing mechanism impacts the network evolutionary topology.

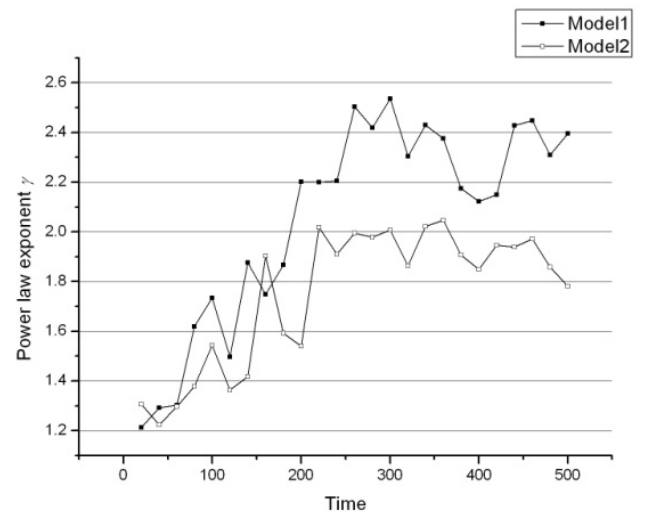

Figure 15. Power law exponent

\section{Summary}

So far, the existing literature about e-commerce market network is very inadequate, and the limitations of traditional methodology result in barriers to deep understanding the evolutionary process of market. In this paper, from a complex network point of view, an analytical framework for e-commerce market networks is presented. We believe that our study will lead to further research that will reveal the hidden 
mechanism of economic and social system and will also enrich the complex network theory.

Based on the above analytical framework and empirical analysis, metabolism and adaptation mechanisms are investigated, and an innovative model is proposed and some original results are obtained.

First, our research reveals that the attraction mechanism impacts evolutionary trend and network structure to some extent. Contrast to the evolutionary trend of degree preferential attachment, the one with attraction preferential attachment reaches a smaller power-law exponent generally. It implies that the majority of relationships are gathered to a few nodes, and the concentration of the network is more obvious.

Second, our research reveals that the global-local-factor and select-order-factor impact the evolutionary structure, and the smaller the probability is, the smaller the concentration of networks is, and the more obvious the randomness is.

Third, the paper reveals that the edge-increasing mechanism makes the network become a small world with a lower average distance and a higher clustering coefficient, and makes it be like a scale-free network with a lower power-law exponent and a higher centralization. In addition, mean of degree, number of nodes, clustering coefficient and density of network in model 2 which has edge-increasing mechanism are obviously higher than these in the contrast model.

\section{REFERENCES}

1. BIANCONI, G., A. L. BARABASI, Competition and Multicaling in Evolving Networks, EPL (Europhysics Letters), vol. 54, no. 4, 2001, pp. 436-442.

2. EASLEY, D., J. KLEINBERG, Networks, Crowds, and Markets, Cambridge: Cambridge University Press, 2010.

3. LAMANAUSKAS, V., V. SLEKIENE, A. BALOG, C. PRIBEANU, Exploring the Usefulness of Social Networking Websites: a Multidimensional Model, Studies in Informatics and Control, vol. 22(2), 2013, pp. 175-184.

4. SCHWEITZER, F., G. FAGIOLO, D. SORNETTE, Economic Networks: The
New Challenges, Science, vol. 325, no. 5939 2009, pp. 422.

5. RAUCH, J. E., Does Network Theory Connect to the Rest of Us? A Review of Matthew O. Jackson's Social and Economic Networks, Journal of Economic Literature, 2010, pp. 980-986.

6. WILKINSON, I., A History of Network and Channels Thinking in Marketing in the 20th Century, Australasian Marketing Journal, vol. 9, no. 2, 2001, pp.23-52.

7. WILKINSON, I., Business Relating Business: Managing Organizational Relations and Networks, Edward Elgar Publishing, 2008.

8. MAURER, S. M., B. A. HUBERMAN, Competitive Dynamics of Web Sites, Journal of Economic Dynamics and Control, vol. 27(11), 2003, pp. 2195-2206.

9. China B2B research centre, 1997-2009 E-commerce investigation report for 12 years in China, 2009.

10. IRESEARCH, 2012 Top 50 of B2C online retailers in China, 2012.

11. CNNIC, 2010 Report of the user research of B2C vertical commodity shopping network in China, 2010.

12. BARABASI, A. L., R. ALBERT, Emergence of Scaling in Random Networks, Science, vol. 286(5439), 1999, pp. 509-512.

13. BANCIU, D., A. G. PITIC, D. VOLOVICI, A. C. MITEA, Using Social Networking Software to Promote Digital Libraries, Studies in Informatics and Control, vol. 21(2), 2012, pp. 221-226.

14. ZHANG, Y., Y. FANG, K. K. WEI, Repurchase Intention in B2C e-Commerce - A Relationship Quality Perspective, Information \& Management, vol. 48(6), 2011, pp. 192-200.

15. CIOCA, M., L. I. CIOCA, L. DUTA, Web Technologies and Multi-criterion Analysis used in Enterprise Integration, Studies in Informatics and Control, vol. 20, no.2, 2011, pp. 129-134. 